\title{
“COLD” BONE SCANS IN ACUTE OSTEOMYELITIS
}

\author{
DONALD C. JONES, ROBERT B. CADY \\ From The Hospital for Sick Children, Toronto, Ontario
}

\begin{abstract}
The diagnosis of acute osteomyelitis is often very difficult during the first 24 to 48 hours. Bone scanning has been a useful adjunct in this diagnosis by demonstrating increased uptake in the area or areas of involvement. Occasionally the pathological area is "cold" on scanning, which may lead to a misdiagnosis. This paper presents three cases demonstrating this unusual finding.
\end{abstract}

Bone scanning has been established as a dependable means of detecting osteomyelitis before radiographic signs appear (Shuler, Aprill and Weiss 1972; Staheli, Nelp and Marty 1972; O'Connor 1974; Shirazi, Rayudu and Fordham 1974; Silva and Harvey 1974; Majd and Frankel 1975; Nelson and Taylor 1978). The usual finding on a positive bone scan is a localised area of increased uptake of the bone-seeking radiopharmaceutical which corresponds to the area of osteomyelitis. Negative bone scans occur occasionally in acute osteomyelitis and even less frequent is the finding of an area of decreased uptake (photon-deficient foci). Reports of "cold" lesions are present in the literature of nuclear medicine (Sy, Westring and Weinberger 1975; Trackler et al. 1976; Handmaker and Leonards 1976; Teates and Williamson 1977) but, unfortunately, not in the orthopaedic literature. We feel that the orthopaedic community should be aware of this phenomenon for two reasons: first, bone scans are being ordered more frequently by orthopaedic surgeons as the facilities for this study are becoming more readily available and, secondly, this finding may not be as rare as previously supposed. Three patients with acute osteomyelitis presenting with cold bone scans were seen and treated by the orthopaedic surgeons at the Hospital for Sick Children in a period spanning less than nine months; these cases are now presented.

\section{CASE REPORTS}

Case 1. An 18-day-old baby girl was noted to have a small red area under the tibial tubercle of the right knee which rapidly enlarged in size. Within 24 hours she had become lethargic and febrile with a swollen erythematous right calf. She was admitted to the intensive care

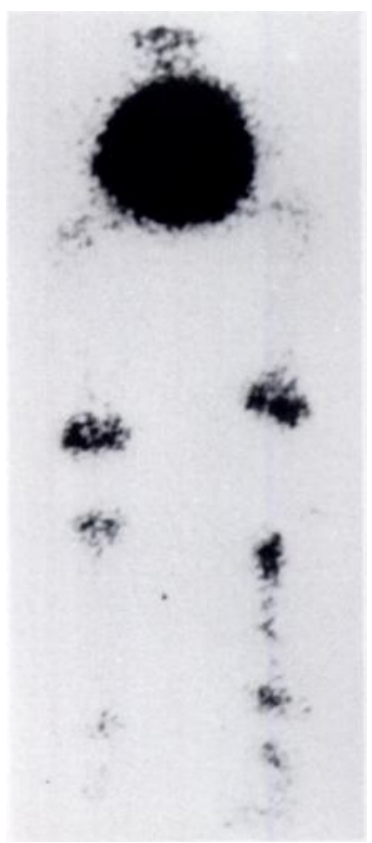

Fig. 1

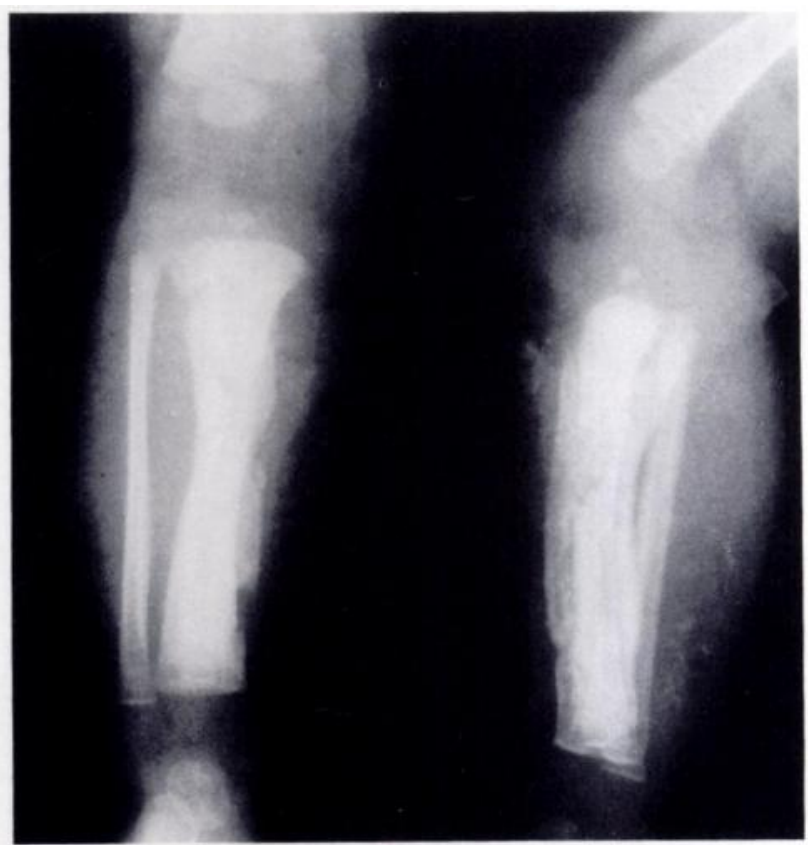

Fig. 2

Case 4 . Figure.1-Decreased uptake in the right tibia. Figure 2-Radiographic changes consistent with acute osteomyelitis of the right tibia.

D. C. Jones, MD, Orthopaedic and Fracture Clinic, 677 East 12th Avenue, Suite 210, Eugene, Oregon 97401, USA

R. B. Cady, MD. Upstate Medical Center, Syracuse, New York, USA.

Requests for reprints should be sent to Dr D. C. Jones.

(c) 1981 British Editorial Society of Bone and Joint Surgery 0301-620X/81/3083-0376 $\$ 2.00$ 
unit of the Hospital for Sick Children with a temperature of 38.6 degrees Celsius, a taut right calf, a white cell count of 5600 cells per microlitre with a normal differential, a prolonged prothrombin time and partial thromboplastin time, and a decreased platelet count Neurologically the limb was normal. Blood cultures were eventually positive for $\beta$-haemolytic streptococcus after intravenous administration of gentamicin, ampicillin and methicillin had been started. The radiographs and venogram were negative. The technetium bone scan revealed a markedly decreased uptake in the right tibia (Fig. 1).

On the fifth day in hospital the right tibia was explored and no purulent material was found although the periosteum from the metaphysial region of the tibia was noted to be stripped off the bone circumferentially. Cultures were negative. The patient improved and

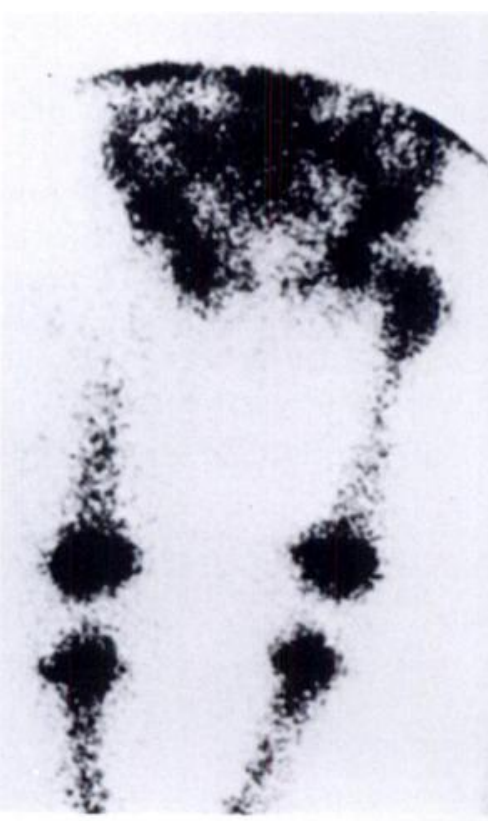

Fig. 3

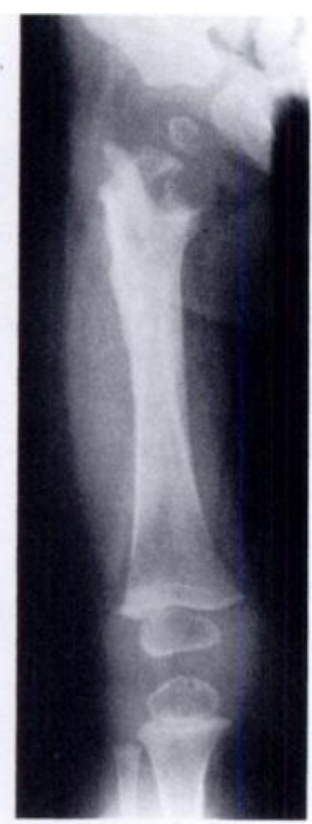

Fig. 4
Case 2. Figure 3-Decreased uptake in the right proximal femur Figure 4-Radiographic changes consistent with osteomyelitis of the right proximal femur. so did the appearance of the leg. She was readmitted two weeks later because of pyrexia and the metaphysis explored again. A bone scan revealed increased uptake and the radiographs demonstrated a large sequestrum (Fig. 2). The bone was drilled revealing pus which grew Staphylococcus aureus.

Case 2. A nine-month-old baby girl developed lethargy and a temperature of 40.6 degrees Celsius without an apparent source of infection. Her erythrocyte sedimentation rate was 110 millimetres in the first hour, her white cell count was 5000 cells per microlitre with a normal differential. Multiple cultures including blood cultures were taken and she was started on ampicillin and kanamycin. She subsequently developed congestive heart failure and generalised convulsions. One blood culture grew Staphylococcus aureus which was resistant to penicillin. Intravenous methicillin was started. Five days after the onset of the illness her right thigh began to swell; radiographs of the femur and hip were normal and a technetium bone scan revealed diminished uptake in the uppermost third of the femur (Fig. 3). As this was thought to represent ischaemic bone, aspiration was performed on the hip, with negative results, and on the proximal femur whence 0.5 millilitres of pus was obtained. The proximal femur was opened and 30 millilitres of subperiosteal pus was found. Subsequent radiographs demonstrated changes consistent with osteomyelitis (Fig. 4).

Case 3. A nine-year-old boy developed acute pain in the right knee without any history of infection or injury. Over the next five days his pain increased and his knee swelled massively. The radiographs proved to be negative and a diagnosis of "housemaid's knee" was made. His temperature increased to 40 degrees Celsius, he was admitted to hospital and started on intravenous antibiotics. After two weeks his fever subsided, the antibiotics were stopped and he was transferred to the Hospital for Sick Children. His temperature again increased to 38.5 degrees Celsius, and his knee remained swollen, warm and diffusely tender without restriction of movement. He had an erythrocyte sedimentation rate of 93 millimetres in the first hour, a white cell count of 7500 cells per microlitre with a slight shift to the left; there was no rheumatoid factor nor antinuclear antibody, and fluid aspirated from the knee showed no growth on culture.

A technetium bone scan revealed decreased uptake in the right patella (Figs 5 and 6 ). The prepatellar bursa was explored surgically and found to be filled with purulent material which had extruded from a cortical defect in the inferior pole of the patella. Cultures from the patella grew Staphylococcus aureus. A bone scan two weeks later demonstrated increased uptake and radiographs showed changes consistent with osteomyelitis (Fig. 7).

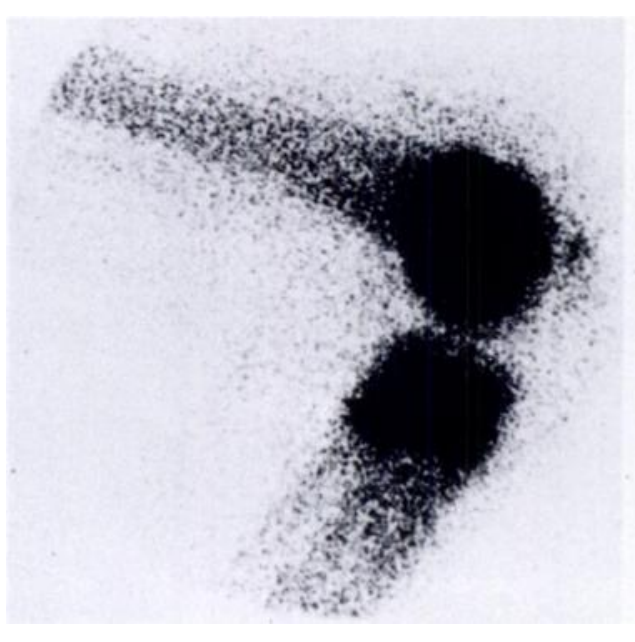

Fig. 5

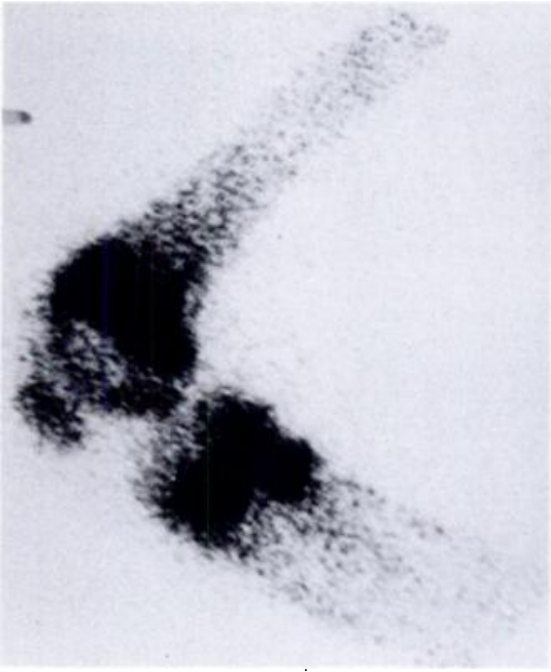

Fig. 6

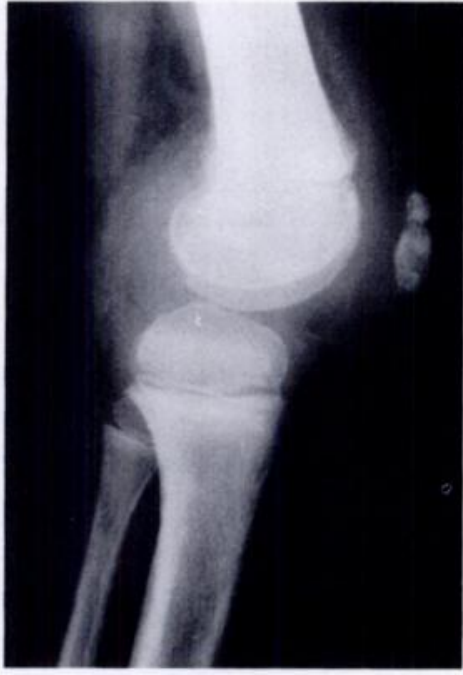

Fig. 7

Case 3. Figure 5-Decreased uptake in the patella of the right knee. Figure 6-Normal uptake in the unaffected left patella. Figure 7-Radiographic changes consistent with acute osteomyelitis of the right patella. 


\section{DISCUSSION}

Acute osteomyelitis is not the only cause of cold lesions in the patient presenting with pain. Bone infarcts in sickle-cell crises, post-traumatic aseptic necrosis, Perthes' disease and metastatic bone disease may give a similar picture (Goergen et al. 1974; Kim, Deland and Maruyama 1978). The presence of these cold defects may pose problems in interpretation of the bone scan and delay treatment. The physician must correlate the clinical history, the physical findings, the laboratory results, the radiographs and the bone scan in making a diagnosis. However, with the awareness that acute osteomyelitis may present as a "cold" lesion instead of the more usual "hot" lesion, the orthopaedic surgeon may promptly administer life-saving antibiotics and undertake the drainage procedures which are indicated.

The exact mechanism of the skeletal uptake of technetium-labelled phosphate remains a mystery. However, it is well recognised that some of the uptake is secondary to adsorption of the radionuclide onto the hydroxyapatite crystals of bone. It is also known that new bone formation and blood flow are important factors in promoting heavy concentration of radionuclide in one area of bone (Kim et al. 1978). Therefore, any mechanism which interferes with hyperaemia or osteoblastic activity is likely to produce a bone image of decreased radionuclide uptake. Theoretically this local ischaemia could be produced by compromise of the microcirculation from subperiosteal or intra-osseous pus (Russin and Staab 1976; Trackler et al. 1976). If the subperiosteal abscess is in the proximity of the nutrient artery it is conceivable that it could cause extrinsic pressure on the artery, resulting in local ischaemia and a subsequent "cold" lesion (Waldvogel, Medoff and Swartz 1970). Unfortunately, there are no clinical arterial studies to prove or disprove this theory.

If in the very early stages of suspected osteomyelitis the routine bone scan should be cold, it has been recommended by Teates and Williamson (1977) that a gallium scan be done. They presented a case in which a 99m Tc-polyphosphate scan demonstrated decreased uptake only to be followed by a "hot" defect on gallium scanning 48 hours later.

In our three cases, as with previously reported cases, the area of involvement changed to an area of increased uptake despite the very early cold scan. It seems likely that this change resulted from spontaneous or surgical relief of pressure by decompression of the intra-osseous or subperiosteal pus, resulting in hyperaemia and the subsequent deposition of increased amounts of radionuclide.

In conclusion, it may be said that a cold bone scan is not inconsistent with acute osteomyelitis and should not delay appropriate therapy.

\section{REFERENCES}

Goergen TG, Alazraki NP, Halpern SE, Heath V, Ashburn WL. "Cold" bone lesions: a newly recognised phenomenon of bone imaging. J Nucl Med 1974;15:1120-4.

Handmaker H, Leonards R. The bone scan in inflammatory osseous disease. Semin Nucl Med 1976;6:95-105.

Kim EE, Deland FH, Maruyama Y. Decreased uptake in bone scans ("cold lesions") in metastatic carcinoma. J Bone Joint Surg [Am] 1978;60-A:844-6.

Majd M, Frankel RS. Bone scanning in osteomyelitis, cellulitis, and bone infarct in children. J Nucl Med 1975;16:547.

Nelson HT, Taylor A. Bone scanning in the diagnosis of osteomyelitis. $J$ Nucl Med 1978;19:696.

O'Connor JF. Radiology and pediatrics. New relationships. Pediatr Clin North Am 1974;21:323-40.

Russsin LD, Staab EV. Unusual bone-scan findings in acute osteomyelitis: case report. J Nucl Med 1976;17:617-19.

Shirazi PH, Rayudu GVS, Fordham EW. ${ }^{18} \mathrm{~F}$ bone scanning: review of indications and results of 1,500 scans. Radiology 1974;112:361-8.

Shuler SE, Aprill CN, Weiss TE. Peripheral joint imaging. Method of evaluation of pediatric bone or joint disease. Am J Dis Child 1972; 4:673-8.

Silva J Jr, Harvey WC. Detection of infections with gallium-67 and scintigraphic imaging. $J$ Infect Dis 1974;130:125-31.

Staheli LT, Nelp WB, Marty R. Strontium 87m scanning. Early diagnosis of bone and joint infections in children.JAMA 1972;221:1159-60.

Sy MW, Westring DW, Weinberger G. "Cold" lesions on bone imaging. $J$ Nucl Med 1975;16:1013-6.

Teates CD, Williamson BRJ. "Hot and cold" bone lesion in acute osteomyelitis. AJR 1977;129:517-8.

Trackler RT, Miller KE, Sutherland DH, Chadwick DL. Childhood pelvic osteomyelitis presenting as a "cold" lesion on bone scan. Case report.J Nucl Med 1976;17:620-2.

Waldvogel FA, Medoff G, Swartz MN. Osteomyelitis: a review of clinical features, therapeutic considerations and unusual aspects. $N$ Engl $J$ Med 1970;282:198-206. 